\title{
MAXIMAL AND MINIMAL POSITIVE SOLUTIONS FOR NONLINEAR RADIATION PROCESSES WITH NONLINEAR SINKS*
}

\author{
By C. Y. CHAN (Florida State University)
}

1. Introduction. Let $D$ be a bounded $\mathbf{n}$-dimensional domain in the real $\mathbf{n}$-dimensional Euclidean space, $D^{-}$its closure, and $\partial D$ its boundary. Also let $x=\left(x_{1}, x_{2}, \cdots, x_{n}\right)$, $\Omega=D \times(0, T], T<\infty, S=\partial D \times(0, T]$, and

$$
B_{\tau}=D \times[0, T] \cap\{t=\tau\} .
$$

The semilinear uniformly parabolic equation under consideration is

$$
L u \equiv \sum_{i, j=1}^{n} \frac{\partial}{\partial x_{i}}\left\{a_{i j}(x, t) \frac{\partial u}{\partial x_{i}}\right\}+c(x, t) u-\frac{\partial u}{\partial t}=g(x, t ; u) \text { in } \Omega,
$$

where $c \leq 0$ is continuous, and $a_{i j}=a_{j i}(i, j=1,2,3, \cdots, n)$ are continuously differentiable. The initial and nonlinear radiation boundary conditions are given respectively by

$$
\begin{gathered}
u(x, 0)=\phi(x) \text { on } B_{0}^{-} \\
\frac{\partial u}{\partial \nu(x, t)}+B(x, t ; u)=f(x, t) \text { on } S,
\end{gathered}
$$

where $\nu$ denotes the outward conormal to $S$. Global uniqueness, existence, upper and lower bounds of positive solutions $u(x, t)$ for this type of problem $((1.1)-(1.3))$ were obtained by Chan [1]; see that paper for further references.

The purpose here is to establish global existence of the maximal and the minimal solutions. They are shown to be the limits of a monotone non-increasing sequence of upper bounds and a monotone non-decreasing sequence of lower bounds respectively. Thus the error involved in using a certain approximate solution can be estimated. The convergence in each case is proved to be uniform. Under the conditions which imply uniqueness of the solution, the rate of convergence of each of the above sequences is shown to be geometrical.

Existence of the maximal and the minimal solutions for a first initial-boundaryvalue problem involving a one-dimensional semilinear heat equation was established by Mlak [2] under the assumption that the corresponding strictly upper and lower solutions exist. He also constructed an example [3] with distinct maximal and minimal solutions. We do not use these kinds of assumptions here, and we adopt a different constructive method for our nonlinear second initial-boundary-value problem (1.1)-(1.3).

2. Maximal and minimal solutions. Let $\partial D \in C^{2+\alpha}$ where $0<\alpha<1$, and let the coefficients $a_{i j}$ together with their partial derivatives $\partial a_{i j} / \partial x_{k}(i, j, k=1,2, \cdots, n)$

* Received June 7, 1973; revised version received September 18, 1973. 
and $c$ be Hölder-continuous of exponent $\alpha$ on $\Omega^{-}$. For the nonlinear sink $g$ and the nonlinear radiation term $B$, we assume that

(i) there exists a non-negative Hölder-continuous function $p(x, t)$ of exponent $\alpha$ on $\Omega^{-}$, and a positive continuous function $q(x, t)$ on $S^{-}$respectively such that for bounded functions $u$ and $v$ on $\Omega^{-}$,

$$
\begin{aligned}
& g(x, t ; u(x, t))-g(x, t ; v(x, t)) \leq p(x, t)[u(x, t)-v(x, t)], \\
& B(x, t ; u(x, t))-B(x, t ; v(x, t)) \leq q(x, t)[u(x, t)-v(x, t)]
\end{aligned}
$$

whenever $u>v$ at the point $(x, t)$;

(ii) $g(x, t ; 0)=0$, and $B(x, t ; 0)=0$;

(iii) if $u>0$ at the point $(x, t)$, then $g(x, t ; u(x, t)) \geq 0$, and $B(x, t ; u(x, t))>0$.

Here we note that $p$ and $q$ can be replaced in particular by appropriate constants. Since we are interested in positive solutions, we use the following definition.

Definition. A solution $M(x, t)(m(x, t))$ of the problem (1.1)-(1.3) is said to be maximal (minimal) if $M(x, t) \geq u(x, t)(m(x, t) \leq u(x, t))$ for any positive solution $u(x, t)$.

The following theorem gives a monotone non-increasing sequence of upper bounds of solutions, and also global existence of the maximal solution.

Theorem 1. If $g, \phi, B$ and $f$ are continuous such that $\phi>0$ on $B_{0}{ }^{-}$and $f>0$ on $S$, then the sequence $\left\{M_{i}(x, t)\right\}$ constructed by $L M_{0}=0$ in $\Omega, M_{0}=\phi$ on $B_{0}^{-}, \partial M_{0} / \partial \nu=f$ on $S$, and for $i=0,1,2, \cdots$,

$$
\begin{gathered}
L M_{i+1}=g\left(x, t ; M_{i}\right)+p\left(M_{i+1}-M_{i}\right) \text { in } \Omega, \\
M_{i+1}=\phi \text { on } B_{0}{ }^{-}, \\
\partial M_{i+1} / \partial \nu+B\left(x, t ; M_{i}\right)+q\left(M_{i+1}-M_{i}\right)=f \text { on } S
\end{gathered}
$$

(where $p$ and $q$ are defined in assumption (i)) is well defined and satisfies

$$
0<M_{i+1} \leq M_{i} \leq M_{0} \leq \underset{B_{0}}{c_{1}(\text { l.u.b. } \phi}+\underset{S^{-}}{\text {l.u.b. } f)} \text { on } \Omega^{-},
$$

where $c_{1}$ is a constant depending on $L$ and $\Omega^{-}$. Furthermore, the problem (1.1)-(1.3) has the maximal solution $M(x, t)$ to which the sequence $\left\{M_{i}\right\}$ converges uniformly.

Proof. The Neumann function $N(x, t ; \xi, \tau)$ of $L v=0$ in $\Omega$ and $\partial v / \partial \nu=0$ on $S$, and the Neumann function of $(L-p) w=0$ in $\Omega$ and $\psi_{a} w \equiv(\partial / \partial \nu+q) w=0$ on $S$ exist. Hence the sequence $\left\{M_{i}\right\}$ is well defined.

If $M_{0} \leq 0$ at some point of $\Omega^{-}$, then by the weak maximum principle, $M_{0}$ attains its non-positive minimum at some point $(x, t)$ on $S$, and hence $\partial M_{0}(x, t) / \partial \nu \leq 0$. This contradicts $f>0$ on $S$. Thus $M_{0}>0$ on $\Omega^{-}$. From Lemma 1 of Chan [1],

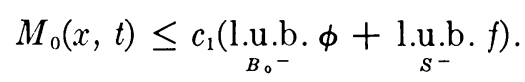

Let us assume that for a particular value of $i$, say $j, M_{j}>0$ on $\Omega^{-}$. Since $g(x, t ; 0)=0$ and $B(x, t ; 0)=0$, it follows from assumption (i) that $g\left(x, t ; M_{i}\right) \leq p M_{i}$, and $B\left(x, t ; M_{i}\right)$ $\leq q M_{i}$. Hence $(L-p) M_{i+1} \leq 0$ in $\Omega$, and $\psi_{a} M_{i+1}>0$ on $S$. Because $M_{i+1}>0$ on $B_{0}^{-}$, we have $M_{i+1}>0$ on $\Omega^{-}$by Lemma 2 of Chan [1]. From the principle of mathematical induction, $0<M_{i}$ on $\Omega^{-}$for $i=0,1,2, \cdots$.

By assumption (iii), $g\left(x, t ; M_{0}\right) \geq 0$ and $B\left(x, t ; M_{0}\right)>0$. Thus 


$$
\begin{aligned}
(L-p)\left(M_{0}-M_{1}\right) & =-g\left(x, t ; M_{0}\right) \leq 0 \text { in } \Omega, \\
\psi_{q}\left(M_{0}-M_{1}\right) & =B\left(x, t ; M_{0}\right)>0 \text { on } S .
\end{aligned}
$$

Since $M_{0}-M_{1}=0$ on $B_{0}{ }^{-}$, it follows from Lemma 2 of Chan [1] that $M_{0} \geq M_{1}$ on $\Omega^{-}$.

Let us assume that for a particular value of $i$, say $j(\geq 1), M_{i} \leq M_{i-1} \leq \cdots \leq M_{0}$ on $\Omega^{-}$. By assumption (i), $(L-p)\left(M_{i}-M_{i+1}\right) \leq 0$ in $\Omega$, and $\psi_{a}\left(M_{i}-M_{i+1}\right) \geq 0$ on $S$. Since $M_{i}-M_{i+1}=0$ on $B_{0}^{-}$, we have from Lemma 2 of Chan [1] that $M_{i} \geq M_{i+1}$ on $\Omega^{-}$. (2.4) then follows from the principle of mathematical induction.

The fact that the sequence $\left\{M_{i}\right\}$ converges to a solution $M$ follows from an argument similar to that in establishing Theorem 6 of Chan [1]. By Dini's theorem (cf. Royden [4, pp. 140-141]), the convergence is uniform.

It remains to be shown that $M$ is the maximal solution. Let $u$ be any positive solution of the problem (1.1)-(1.3). Then $g(x, t ; u) \geq 0, B(x, t ; u)>0$, and we have $L\left(u-M_{0}\right) \geq 0$ in $\Omega, u-M_{0}=0$ on $B_{0}{ }^{-}$, and $\partial\left(u-M_{0}\right) / \partial \nu<0$ on $S$. If $u>M_{0}$ at some point on $\Omega^{-}$, then $u-M_{0}$ attains its positive maximum at some point $(x, t)$ of $S$ so that $\partial\left(u-M_{0}\right) / \partial \nu \geq 0$, and we have a contradiction. Thus $u \leq M_{0}$ on $\Omega^{-}$.

Let us assume that $M_{i} \geq u$ on $\Omega^{-}$for some $j$. By assumption (i), $(L-p)\left(M_{i+1}-u\right)$ $\leq 0$ in $\Omega$, and $\psi_{q}\left(M_{i+1}-u\right) \geq 0$ on $S$. On $B_{0}{ }^{-}, M_{i+1}-u=0$. Therefore from Lemma 2 of Chan [1], $M_{j+1} \geq u$ on $\Omega^{-}$. That $M$ is the maximal solution follows from the principle of mathematical induction.

The following theorem gives a monotone non-decreasing sequence of lower bounds of solutions, and also global existence of the minimal solution. Since its proof is similar to that of Theorem 1, we omit it here.

Theorem 2. Under the hypotheses of Theorem 1 , the sequence $\left\{m_{i}(x, t)\right\}$ constructed by $m_{0} \equiv 0$ on $\Omega^{-}$, and for $i=0,1,2, \cdots$,

$$
\begin{gathered}
L m_{i+1}=g\left(x, t ; m_{i}\right)+p\left(m_{i+1}-m_{i}\right) \text { in } \Omega, \\
m_{i+1}=\phi \text { on } B_{0}^{-}, \\
\partial m_{i+1} / \partial \nu+B\left(x, t ; m_{i}\right)+q\left(m_{i+1}-m_{i}\right)=f \text { on } S
\end{gathered}
$$

is well defined and satisfies

$$
0<m_{i} \leq m_{i+1} \leq M_{0} \quad(i=1,2,3, \cdots) \quad \text { on } \Omega^{-} .
$$

Furthermore, the problem (1.1)-(1.3) has the minimal solution $m(x, t)$ to which the sequence $\left\{m_{i}\right\}$ converges uniformly.

That the maximal solution is positive follows from its definition. Because of (2.5), we have the following corollary.

Corollary 1. Under the hypotheses of Theorem 1, the minimal solution of the problem (1.1)-(1.3) is positive on $\Omega^{-}$.

In each step of the above constructions of the maximal and the minimal solutions, the same Neumann function of $(L-p) w=0$ in $\Omega$ and $\psi_{a} w=0$ on $S$ is used, except in the initial step of constructing $M_{0}$ when we use the Neumann function $N(x, t ; \xi, \tau)$. Our next theorem shows that under the additional conditions which guarantee uniqueness of the solution, the rate of convergence of each of the sequences $\left\{M_{i}\right\}$ and $\left\{m_{i}\right\}$ to the solution is geometrical.

Theorem 3. Under the hypotheses of Theorem 1, if 


$$
\begin{array}{lll}
g(x, t ; u(x, t)) \geq g(x, t ; v(x, t)) & \text { when } \quad u(x, t)>v(x, t), \\
B(x, t ; u(x, t))>B(x, t ; v(x, t)) & \text { when } \quad u(x, t)>v(x, t),
\end{array}
$$

then each of the sequences $\left\{M_{i}\right\}$ and $\left\{m_{i}\right\}$ converges uniformly and geometrically to the unique solution $\lim _{i \rightarrow \infty} M_{i}=\lim _{i \rightarrow \infty} m_{i}$ of the problem (1.1)-(1.3).

Proof. Under assumptions (2.6) and (2.7), it follows from Corollary 1 of Chan [1] that there exists at most one solution to the problem (1.1)-(1.3). From Theorems 1 and 2 , this unique solution is given by $\lim _{i \rightarrow \infty} M_{i}=\lim _{i \rightarrow \infty} m_{i}$, and in each case the convergence is uniform.

To show that the sequence $\left\{M_{i}\right\}$ converges geometrically, we use Green's identity to rewrite the iteration scheme (2.1)-(2.3) equivalently as

$$
\begin{aligned}
M_{i+1}(x, t)= & \int_{B_{0}} N(x, t ; \xi, 0) \phi(\xi) d V_{\xi} \\
& -\int_{0}^{t} \int_{B_{\tau}} N(x, t ; \xi, \tau)\left[g\left(\xi, \tau ; M_{i}\right)+p\left(M_{i+1}-M_{i}\right)\right] d V_{\xi} d \tau \\
& +\int_{0}^{t} \int_{\partial B_{\tau}} N(x, t ; \xi, \tau)\left[f(\xi, \tau)-B\left(\xi, \tau ; M_{i}\right)-q\left(M_{i+1}-M_{i}\right)\right] d A_{\xi} d \tau .
\end{aligned}
$$

Since $M_{i} \geq M_{i+1}$ on $\Omega^{-}$, and $N(x, t ; \xi, \tau)>0$ in $D \times(\tau, T]$ for each $(\xi, \tau)$ in $D \times[0, T)$, it follows from (2.6) and (2.7) that

$$
\begin{aligned}
M_{i}-M_{i+1} \leq & \int_{0}^{t} \int_{B_{\tau}} p N(x, t ; \xi, \tau)\left(M_{i-1}-M_{i}\right) d V_{\xi} d \tau \\
& +\int_{0}^{t} \int_{\partial B_{\tau}} q N(x, t ; \xi, \tau)\left(M_{i-1}-M_{i}\right) d A_{\xi} d \tau
\end{aligned}
$$

after having dropped out the non-positive terms on the right-hand side. Let

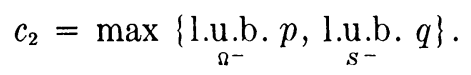

Then

$$
\begin{aligned}
M_{i}-M_{i+1} \leq & c_{2}\left[\int_{0}^{t} \int_{B_{\tau}} N(x, t ; \xi, \tau)\left(M_{i-1}-M_{i}\right) d V_{\xi} d \tau\right. \\
& \left.+\int_{0}^{t} \int_{\partial B_{\tau}} N(x, t ; \xi, \tau)\left(M_{i-1}-M_{i}\right) d A_{\xi} d \tau\right] .
\end{aligned}
$$

An argument similar to the proof of Theorem 4 of Chan [1] establishes the geometrical rate of convergence. Similarly, we can show that the sequence $\left\{m_{i}\right\}$ also converges geometrically.

\section{REFERENCES}

[1] C. Y. Chan, Positive solutions for nonlinear parabolic second initial boundary value problems, Quart. Appl. Math. 31, 443-454 (1974)

[2] W. Mlak, Parabolic differential inequalities and Chaplighin's method, Ann. Polon. Math. 8, 139-153 (1960)

[3] W. Mlak, An example of the equation $u_{t}=u_{x x}+f(x, t, u)$ with distinct maximum and minimum solutions of a mixed problem, Ann. Polon. Math. 13, 101-103 (1963)

[4] H. L. Royden, Real analysis, Macmillan, N. Y., 1963, pp. 140-141 